\title{
Analysis of Diesel Spray Droplets Behavior Using Shadowgraph Technique Images
}

\author{
Abdullah Adam, Mamat Rizalman, and Tomoaki Yatsufusa
}

\begin{abstract}
This study investigates the effects of injection pressure and ambient temperature on distribution of number density and diameter of fuel droplet in diesel fuel spray by using shadow-graph photography technique. The large number of fuel droplets was statistically analyzed by newly developed algorithm. The results show that the larger-size particles exist closer to the spray tip. Higher injection pressure promotes atomization and high ambient temperature has great effect on formation of small-size droplet at early time of injection period, especially at up and midstream of the spray.
\end{abstract}

Index Terms-Atomization, spray images, shadowgraph, droplets measurement.

\section{INTRODUCTION}

Diesel engines have better engine performance, low fuel consumption and greater power output compared to gasoline spark ignition engines. However, diesel engines are still emitting a lot amount of NOx and Particulate Matter at significantly higher level than gasoline engine [1]-[3]. To adhere to regulations related to $\mathrm{CO}_{2}$ emissions, the major green-house gas that causes global warming, studies in search of alternatives to using diesel, along with the development of relevant technologies are needed urgently in order to improve engine performance and to preserve the environment.

In order to produce low emission in diesel engines, combustion process playing the main role. Diesel fuel injection process will dynamically control spray behavior which is known to be a strong parameter that affects combustion process [4]-[6]. The spray behavior and atomization characteristics of diesel spray have been investigated by [7], [8]. They conclude that the knowledge of the fuel spray atomization mechanism can be a key issue for a successful simulation of all the subsequent process of mixture formation and eventually combustion and pollutant formation. On top of that, several researches about numerical and experimental study on fuel atomization of diesel injection spray have also been published [9], [10]. Lee et al. [11] for example, investigated the macroscopic spray structure and the atomization characteristics of high pressure common rail type fuel injection system. They reported that at the downstream of the spray, the droplets positioned

Manuscript received January 8, 2014; revised May 9, 2014.

Abdullah Adam and Mamat Rizalman are with the Automotive Engineering Centre, Pahang, Malaysia (e-mail: adam@ump.edu.my, rizalman@ump.edu.my).

Tomoaki Yatsufusa is with Hiroshima Institute of Technology, Hiroshima, Japan (e-mail: t.yatsufusa.nr@cc.it-hiroshima.ac.jp). outside of the main spray are disintegrated into the small particles prior to the inside droplets because of the large relative velocity between spray and ambient gas.

In this research, analysis of direct photographic imaging has been done thoroughly to quantify a set of macroscopic, characteristic parameters of the sprays including tip penetration, near-and far-field angle. To do so, dedicated image processing software has been developed to quantify a set of macroscopic, characteristic parameters of the sprays including tip penetration, near-and far-field angles. The results obtained from image analysis under various spray conditions demonstrate that the software is capable of producing accurate, consistent and efficient results.

\section{EXPERIMENTAL APPARATUS}

The experimental apparatus shown in Fig. 1 consists of a spray chamber, a rapid compression machine, a single-shot common rail injection system and a nano-spark shadow graph photography unit. The diesel spray was injected in a constant volume spray chamber to stimulate real diesel engine combustion process. The spray chamber diameter is $60 \mathrm{~mm}$ and a width $20 \mathrm{~mm}$. $\mathrm{N}_{2}$-Argon gas mixture was supplied into spray chamber replacing the air inside spray chamber. The gas mixture function is to prevent the diesel fuel from ignite thus enable the photography of the evaporating spray [12]. The induced gas density inside the spray chamber was kept constant at $15 \mathrm{~kg} / \mathrm{m}^{3}$, which simulated gas density inside the real diesel engine cylinder at start of injection. The ambient pressure at start of injection was recorded at $p_{i}=1 \mathrm{MPa}$ and $2.4 \mathrm{MPa}$ at ambient temperature of $T_{i}=298 \mathrm{~K}$ and $T_{i}=700 \mathrm{~K}$ respectively.

A single-shot common rail fuel injection system was used to inject the fuel into the spray chamber. Injection nozzle was a single-hole nozzle with hole-diameter of $d_{n}=0.18 \mathrm{~mm}$. The injection pressure varies from $P_{i n j}=40 \mathrm{MPa}$ to $100 \mathrm{MPa}$. The injection period was fixed constant at $2.0 \mathrm{~ms}$ and diesel fuel JIS\#2 (density $836 \mathrm{~kg} / \mathrm{m}^{3}$ ) was used in the experiment.

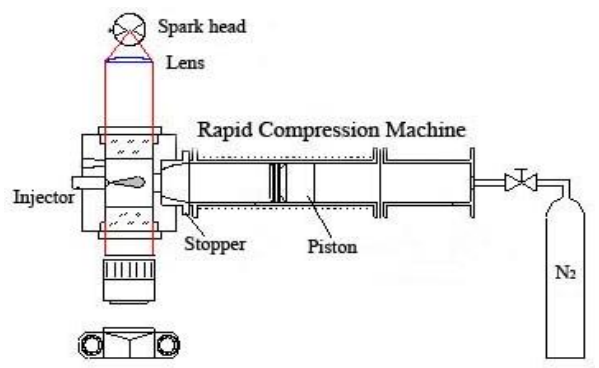

Fig. 1. A rapid compression machine and shadow-graph system.

The first stage of the processing algorithm is to extract 
droplets image from background images. Fig. 2 shows original TIFF image and its binary image of droplets. Threshold level for processing binary image was acquired following below mathematical equation:

\section{Threshold $=$}

$\left(\right.$ Avg_intensity $\left.-M i n \_i n t e n s i t y\right) \times \alpha+$ Min_intensity

Referring to equation (1), Avg_intensity represents average intensity value throughout the image while Min_intensity represents minimum intensity value for the image. Coefficient $\alpha$ value was set at 0.8 . This value was selected based on the outcome of the binary image. From the binary image, location of the possible droplets images can be detected and an algorithm will analyze the images to acquire the intensity gravity center of each of the possible droplets image. In this algorithm, the different levels of gradient intensity of droplets image was utilize for droplets position and size detection.

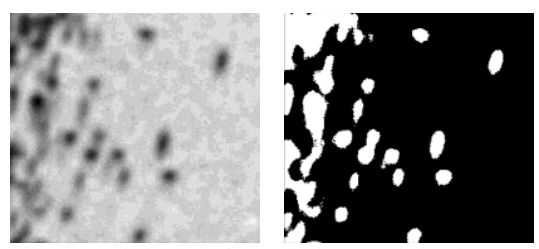

Fig. 2. Original (left) and binary (right) image.

Each droplet images extracted from previous step will be further analyzed to confirm whether it can be accepted as droplet images or to be excluded. Fig. 3 shows from upper left, intensity profiles of TIFF image and its droplet TIFF image and from lower left, gradient intensity profiles of gradient image and its droplet gradient image. Droplets gravity center was first acquired by the program and then, the program will calculate intensity value at 50 pixel from center point toward outside of the center gravity as shown in Fig. 4.

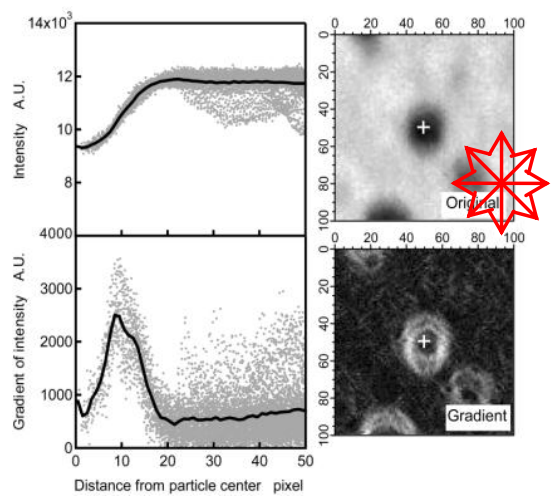

Fig. 3. Intensity and gradient intensity profiles of regular droplets.

Fig. 4 shows gradient profile of a single droplets image. From the graph, highest peak of gradient intensity shows maximum intensity value for the image while average intensity value can be acquired by calculating the total intensity value measured throughout 50 pixel projection from gravity center. Mid Point can be acquired by selecting the middle point of the gradient intensity distribution value which above the average intensity. Furthermore, valuable information acquire from the gradient intensity profile are minimum intensity (Min_IN) at center image, lowest intensity (Min_OUT).

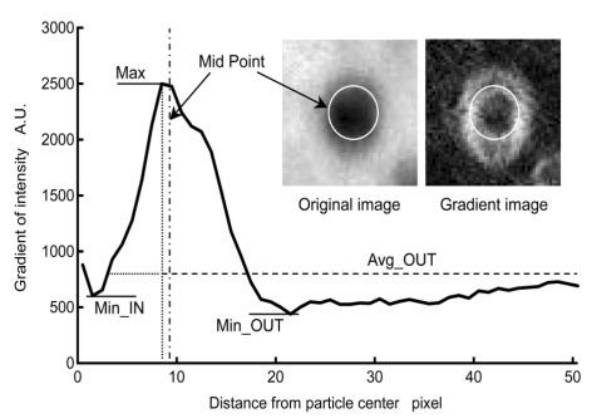

Fig. 4. Details of intensity gradient profiles.

During the droplets image analyzed process, not all the droplets images acquired are in fine round shape as shown in Fig. 4. In some cases, irregular droplets images as shown in Fig. 5 can frequently be found during the analysis process. Therefore, these droplets images must be further analyzed before it can be accepted as droplets image. Below shows equations applied in this algorithm to confirm the gradient intensity shape was within the acceptable value as a droplet or should be excluded from analysis.

$$
\begin{gathered}
\text { Ratio_IN }=\frac{M a x_{-} I N T-M i n_{-} I N}{M a x_{-} I N T-M i n_{-} O U T} \\
\text { Ratio_OUT }=\frac{A v g_{-} O U T-M i n}{M a x_{-} I N T-M i n}
\end{gathered}
$$

Here, Min represents lowest intensity either Min_IN or Min_OUT. From above equation, shape of the gradient intensity peak can be determined. Large value of Ratio_IN or small value of Ratio_OUT shows that gradient intensity shape has high peak intensity value profiles and will be accepted as a droplet image by the algorithm. Meanwhile, small value of Ratio_IN or large value of Ratio_OUT shows the gradient intensity shape has no high peak and will not be selected by algorithm for further analysis.

Pre-experiment was done to collect comparison in order to build an algorithm which can measured spray droplets size inside spray images. Accordingly, similar size of sphere plastics particle was placed on the thin glass and was measured by using laboratory microscope. These plastic particle images were captured at magnification factor of 4 by microscope and by Pentax $67135 \mathrm{~mm}$ lens at actual experiment setting.

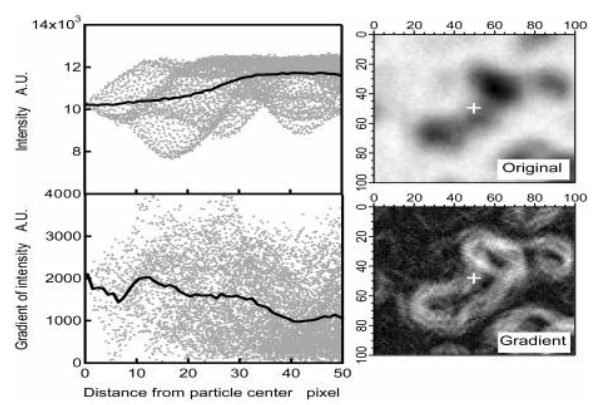

Fig. 5. Intensity and gradient intensity profiles of irregular droplets.

Fig. 6 shows sample of comparison images of plastic 
particle taken with microscope (left) and experiment setup (right). Plastic particle images taken by microscope show clear sphere images and sharp edge while images taken by Pentax 67 135mm lens shows blurred dark images where the edges were not sharp and gradiently blend with the background.

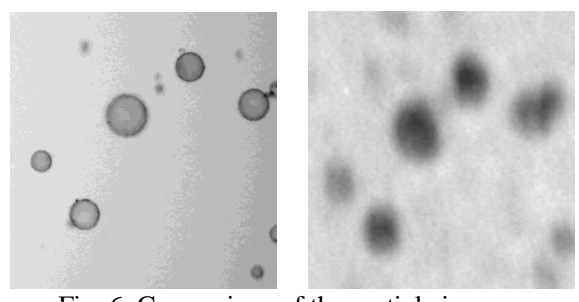

Fig. 6. Comparison of the particle images.

Fig. 7 shows correlation between Mid Point of each particle image and actual diameter of particle measured with microscope images and lens images. Referring to Fig. 7, scattered data correlation can be seen forming a curve pattern inside graph. However, at the same time, many unwanted data was picked up and measured show the algorithm was not reliable. Therefore, Mid Point calculation data must be stringent and the unwanted data must be removed in order to increase the accuracy of the program algorithm so the algorithm becomes reliable for fuel droplets measurement. As a result, any data which was not fulfill the acceptable value of 0.6 above for Ratio_IN, less than 0.3 for Ratio_OUT and less than 1.5 pixel for prdev will be exclude from analysis and was not considered as droplets.

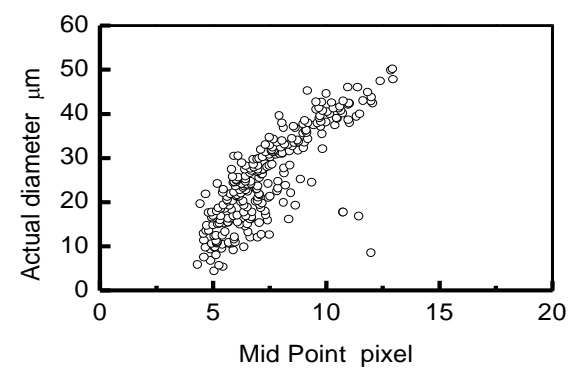

Fig. 7. Correlation between Mid Point and actual diameter of particles filtered by Ratio_IN, Ratio_OUT, and prdev.

The re-calculation result was shown in Fig. 8. Data correlation formed a detectable curve line thus and the unwanted data were excluded from the result.

The examples of data pick up and elimination of miss detected particles was shown in Fig. 9. Referring to the figure, clear shape and individual particles were picked up inside box while unclear shape and daubs particle at right upper edge of images was exclude from analysis.

Since the actual spray is in three dimension geometry, changes in term of the droplets/particle characteristics as it becomes out of focus are among an important factor to be considered in the algorithm. Accordingly, the in-focus particle images appears to be sharp and clear while out of focus particle images appears to be blur and hard to recognized. When particle are inside the focal depth region, its image has a high image intensity gradient around the image boundary thus it has a clear image. However, when it is not inside the focal depth region, its image intensity decreases and unclear image can be seen on the photograph. The effect of particle position with regards to the focal depth on particle images was examined using the algorithm and the result is shown in Fig. 10.

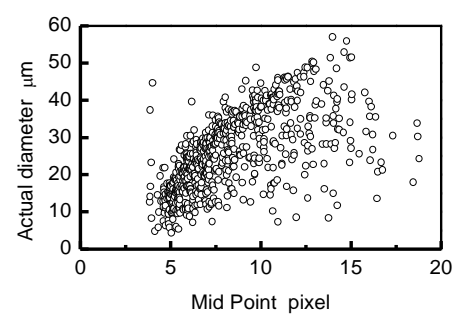

Fig. 8. Correlation between Mid Point and actual diameter of particles.

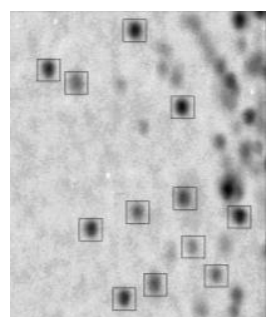

Fig. 9. Example of the elimination of miss-detected particles.

As shown in Fig. 10 particles were set at position of $0.5 \mathrm{~mm}, 1.5 \mathrm{~mm}$ and above $1.5 \mathrm{~mm}$ offset from focal point (center of focal depth region). The correlation results shows that at $0.5 \mathrm{~mm}$ offset from focal point, Mid Point measurement value are leaning toward actual particle diameter and shows that at $0.5 \mathrm{~mm}$ offset from focal point, the algorithm measurement still has high accuracy and reliable. Meanwhile, at $1.5 \mathrm{~mm}$ offset from focal point, Mid Point measurement shows larger value compared to actual diameter size. As a result, the number of particles selected for analysis by the algorithm also reduced thus lowered the accuracy to the measurement. Meanwhile, particles position at above $1.5 \mathrm{~mm}$ offset from focal point were considered not reliable for analysis and successfully not selected by the algorithm.

Referring to Fig. 10, correlation between Mid Point particle measurement and actual particle diameter measured by the algorithm formed a stable curve line at large size particles (on top) while scattered and unsteady curve line was formed at smaller size particles area (bottom). Moreover, at large Mid Point value of 12-13pixel include small range of particles size from $45-50 \mu \mathrm{m}$ where at small Mid Point value of 5-6pixel include large range of particles size from $4-25 \mu \mathrm{m}$. This reveals that small size particles measurement has tendency to increase the measurement error.

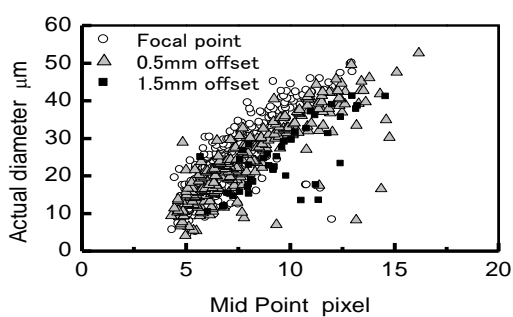

Fig. 10. Effect of deviation from focal point on correlation Mid Point and actual diameter of particles.

Fig. 11 shows error ratio versus actual diameter graph plotted using specifically small Mid Point value of 4-5 pixel and 5-6 pixel which concentrate on small size particles. 
Diameter $10 \mu \mathrm{m}$ particles show a measurement error ratio at almost 40. The error was too big and cannot be accepted in the algorithm. However, in order to reduce error, the number of particles selected for analysis must be increased. Right side of the graph in Fig. 11 shows minimum particle number needed in order for the error ratio to be less than $5 \%$. As a results, as shown by $\triangle$ symbol inside Fig. 11, more than 60 numbers of particles must be selected in case of small particle size around $10 \mu \mathrm{m}$ in order to reduce the error ratio at $5 \%$.

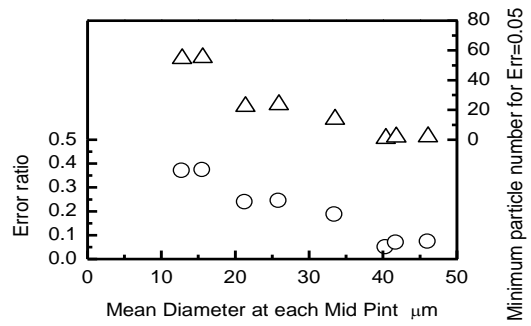

Fig. 11. Relation between accuracy of particle diameter measurement and particle diameter.

\section{RESUlTS AND DisCUSSION}

In order to further analyze the behavior of the droplets, 2 set of spray condition were employed in this research. Fig. 12 shows sample of full spray images and detail images taken at $P_{i n j}=40 \mathrm{MPa}, 70 \mathrm{MPa}$ and $T_{i}=298 \mathrm{~K}, 700 \mathrm{~K}$. Both spray images were taken at $t=0.5 \mathrm{~ms}$. The spray images categorized as i) High fuel injection pressure $P_{i n j}$ condition and ii) High ambient temperature $T_{i}$ condition. Table I shows the parameter setup for spray images and the total number of droplets recognized by the algorithm. From table obviously the lowest number of droplets spotted in high ambient temperature condition.

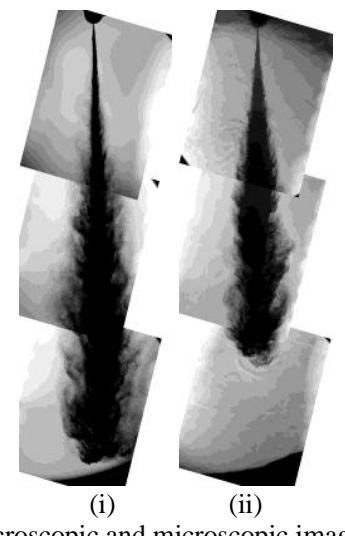

Fig. 12. Macroscopic and microscopic images of sprays

Fig. 13 shows analysis of droplets frequency at each spray condition. The analysis was done based on the observation area of upstream (UP), middle (MD) and downstream (DW) areas. Since the algorithm accuracy dropped in case of small size droplets as shown in Fig. 11, it is crucial to do analysis on large numbers of droplets to validate the results. High $P_{i n j}$ condition spray shows that thin and long spray formed at UP area while larger and thicker spray formed at MD and DW area. Spray penetration length at high $P_{i n j}$ condition marked $30 \%$ longer than high ambient temperature $T_{i}$ condition although the photograph timing $t=0.5 \mathrm{~ms}$ is same for both spray images. The increase of spray momentum for high injection pressure assists the spray to travel with less restriction inside spray chamber. Meanwhile, observation on high ambient temperature spray shows shorter spray penetrations and thin cone angle formed at UP. Overall spray was thicker and number of structures like branches reduce instantly due to the spray atomization process was promotes in high ambient temperature spray.

\begin{tabular}{|c|c|c|}
\hline \multicolumn{3}{|c|}{ TABLE I: EXPERIMENTAL CONDITIONS } \\
\begin{tabular}{|c|c|c|}
\hline & High $P_{i n j}$ & High $T_{i}$ \\
\hline Ambient temperature [K] & 298 & 700 \\
\hline Fuel injection pressure [MPa] & 70 & 40 \\
\hline Image timing [ms] & 0.50 & 0.50 \\
\hline Analyzed particle number & 2232 & 411 \\
\hline
\end{tabular}
\end{tabular}

Spray images were analyzed using the algorithm and the results were plotted and shown in Fig. 13 for i) high fuel injection pressure $P_{i n j}$ condition and Fig. 14 for ii) high ambient temperature $T_{i}$ condition.

Referring to Fig. 13, high injection condition, UP area shows large existence of below $10 \mathrm{~mm}$ small size droplets compare to MD and DW areas. Overall, the number of small size droplets is increase at all area of UP, MD and DW in high injection pressure condition. Therefore, recent common rail fuel injection system that enable the diesel fuel injected at higher injection pressure spray can increase small size droplets along the spray boundary from nozzle to spray tip thus, will improve fuel atomization process.

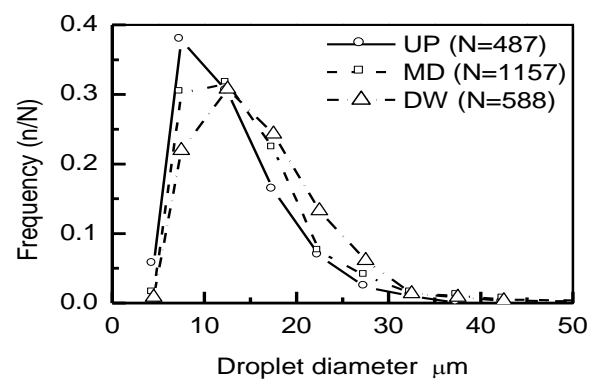

Fig. 13. Particle diameter distribution in high $\mathrm{P}_{\text {inj }}$ spray.

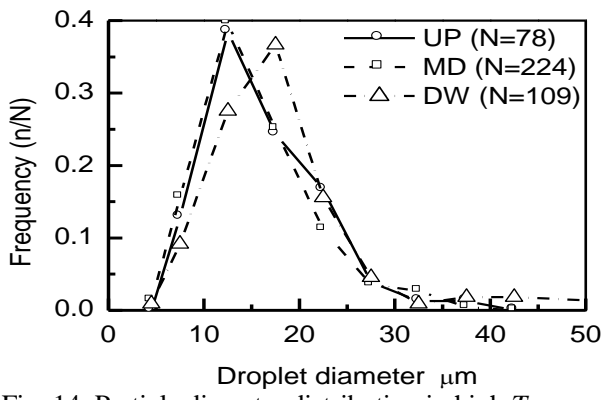

Fig. 14. Particle diameter distribution in high $T_{i}$ spray.

Fig. 14 shows, at high ambient temperature $T_{i}$ condition, the UP and MD peak position shifted to right show compare to high injection pressure spray shows relatively droplets size larger than $12 \mu \mathrm{m}$ exist in this area. The results show that small size droplets evaporate instantly in high ambient temperature spray. Large size droplets exist in the DW area. During the spray penetrates into gas ambient at high velocity and high density of fuel, droplets were produced along the process. Droplets at UP and MD area stay long enough for them to undergo vaporization process thus droplets detected in those areas measured smaller than DW area. Large 
number of small droplets exist in MD could promotes atomization process thus trigger fuel ignition to occurs at this area.

\section{CONCLUSIONS}

This study investigates the effects of injection pressure and ambient temperature on distribution of number density and diameter of fuel droplet in diesel fuel spray by using shadow-graph photography technique. The large number of fuel droplets was statistically analyzed by newly developed algorithm.

1) Large size droplets can easily be detected and measured by using the algorithm. However, the algorithm shows decent error on diameter less than $10 \mu \mathrm{m}$ small size droplets. To increase accuracy, large number of small size droplets need to be detected to increase the measurement accuracy.

2) High injection pressure spray produces large number of small size droplets along its spray boundary especially at UP and MD area while high ambient temperature spray cause small size droplets to vaporize instantly.

3) Combination of high injection pressure spray and high ambient temperature spray could generates small size droplets, expedite vaporization and improve atomization process thus better fuel combustion.

\section{ACKNOWLEDGMENT}

The authors would like to acknowledge the support of the Power Engine Lab, University of Tokushima and Automotive Engineering Centre, University Malaysia Pahang. This work was partly supported by Research Acculturation Grant Scheme (RAGS) RDU121418.

\section{REFERENCES}

[1] J. Oh, K. Lee, and H. Jeong, "Study on the spray behavior and diesel fuel distribution characteristics of a secondary injector for a lean NOx trap catalyst," Energy \& Fuels, vol. 22, pp. 1527-1534, 2008.

[2] Z. Liu, Y. Ge, J. Tan, C. He et al., "Impacts of continuously regenerating trap and particle oxidation catalyst on the $\mathrm{NO}_{2}$ and particulate matter emissions emitted from diesel engine," Journal of Environmental Sciences, vol. 24, pp. 624-631, 2011.

[3] K. Kinoshita, T. Morimune, and S. Goto, "Study on removal of PM and NOx in diesel exhaust by using DC corona discharge," SAE Paper, pp. 01-1660, 2002.

[4] K. K. Song, S. C. Sim, B. K. Jung, H. G. Kim, and J. H. Kim, "Effect of the injection parameters on diesel spray characteristics " Journal of Mechanical Science and Technology, vol. 19, pp. 1321-1328, 2005.

[5] A. Mohammadi, Y. Kidoguchi, and K. K.Miwa, "Effect of injection parameters and wall-impingement on atomization and gas entrainment processes in diesel sprays," SAE Paper, 2002.

[6] X. Wang, Z. Huang, O. A. Kuti, W. Zhang, and K. Nishida, "Experimental and analytical study on biodiesel and diesel spray characteristics under ultra-high injection pressure," International Journal of Heat and Fluid Flow, vol. 31, pp. 659-666, 2010.

[7] S. Hossainpour and A. R. Binesh, "Investigation of Fuel Spray Atomization in a DI heavy-duty diesel engine and comparison of various spray breakup models," Fuel, vol. 88, pp. 799-805, 2009.

[8] S. H. Park, H. J. Kim, and C. S. Lee, "Numerical investigation of combustion and exhaust emissions characteristics based on experimental spray and atomization characteristics in a compression ignition diesel engine," Energy \& Fuels, vol. 24, pp. 2429-2438, 2010.

[9] H. J. Kim, S. H. Park, and C. S. Lee, "A study on the macroscopic spray behavior and atomization characteristics of biodiesel and dimethyl ether sprays under increased ambient pressure," Fuel Processing Technology, vol. 91, pp. 354-363, 2010.

[10] E. Delacourt, B. Desmet, and B. Besson, "Characterisation of very high pressure diesel sprays using digital imaging techniques," Fuel, vol. 84 , pp. 859-867, 2005

[11] C. S. Lee and S. W. Park, "An experimental and numerical study on fuel atomization characteristics of high-pressure diesel injection sprays," Fuel, vol. 81, pp. 2417-2423, 2002.

[12] A. Adam, N. Inukai, Y. Kidoguchi, and K. M. S. Miyashiro, "A study on droplets evaporation at diesel spray boundary during ignition delay period," SAE Paper, 2007.

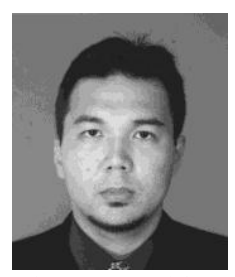

Abdullah Adam was born in Kuantan, Pahang, Malaysia, in 1971. He received the B.E. degree in mechanical engineering from the University of Akita, Japan in 1995, and the M.Eng. and Ph.D. degrees in ecosystem engineering from the University of Tokushima, Japan in 2007 and 2010, respectively.

In 2008, he joined the Faculty of Mechanical Engineering, University Malaysia Pahang (UMP), as a lecturer, and in 2010 became a senior lecturer. Since July 2011, he was appointed as the director of Automotive Engineering Centre. His current research interests include diesel spray and combustion, biodiesel and biomass fuel, engine performance and emission. Dr. A. Adam is a member of Society of Automotive Engineers (SAE), Japan Society of Automotive Engineers and Malaysian Society of Engineering and Technology. 